\title{
SOLUTIONS OF A NONLINEAR PARTIAL DIFFERENTIAL EQUATION OF HYPERBOLIC TYPE*
}

\author{
BY \\ C. E. BILLIGHEIMER \\ University of Toronto
}

I. Introduction. We discuss in this paper solutions of the equation

$$
\square^{2} u=b u+c u^{2}
$$

where

$$
\square^{2}=\frac{\partial^{2}}{\partial x^{2}}+\frac{\partial^{2}}{\partial y^{2}}+\frac{\partial^{2}}{\partial z^{2}}-\frac{1}{c^{2}} \frac{\partial^{2}}{\partial t^{2}}
$$

(1.1) is a nonlinear partial differential equation of hyperbolic type, which may be regarded physically as the Klein-Gordon equation of a neutral scalar meson field with added nonlinear term $c u^{2}$.

Nonlinear interaction terms have been introduced into the meson field equations in considering such problems as the saturation of nuclear forces (Thirring 1953 (1)) or the production of mesons in the collision of high energy nucleons (Heisenberg 1952, Schiff 1951). Further reasons in favour of such a procedure are summarized by Cap (1956). The nonlinearity introduces considerable complication into the mathematical treatment. Various authors (Hurst 1952, Petermann 1953, Thirring 1953 (2), Utiyama and Imamura 1953) have proved the divergence of perturbation expansions in a qu mium field theory with nonlinearity of the type we are considering, and renormalizatic does not remove this divergency (Thirring 1953 (2)).

The classical discussion of solutions of the nonlinear wave equation (1.1) itself seems of interest. We are particularly interested in bounded solutions, and it appears from the special cases studied that, for example in the case of the initial value prob$\mathrm{lem}$, bounded solutions exist for sufficiently bounded initial conditions. For larger initial values of the function and appropriate differential coefficients say, we find that the solutions become unbounded after a finite time.

We discuss in this paper the special cases of solutions of Eq. (1.1) that are obtainable by solving an ordinary differential equation.

In Scc. I we obtain all possible cases in which Eq. (1.1) reduces to an ordinary differential equation in the one-dimensional case, as well as some analogous circularly and spherically symmetric cases in two and three dimensions respectively.

In Secs. 2 and 3 we then discuss in turn the solution behaviour of the two main types of equation obtained, the cases $a=\frac{1}{2}$ and $a>\frac{1}{2}$ of an equation of the form

$$
s u^{\prime \prime}+a u^{\prime}+\bar{b} u+\bar{c} u^{2}=0 .
$$

1. Solutions satisfying an ordinary differential equation. We study in this section ("ases in which the solutions of Ea. (1.1) are obtained by solving an ordinary nonlinear differential equation.

*Received April 12, 1965; revised manuscript received June 13, 1966. 
We discuss first in Sec. 1.1 some fundamental invariance properties of Eq. (1.1). In Sec. 1.2 we obtain all possible ordinary differential equations in the one-dicensional case, and extend these solutions to the case of two and three dimensions by the use of the invariance properties of Sec. 1.1. In Sec. 1.3 we obtain ordinary differential equ tions for some analogous circularly and spherically symmetric cases in two and three dimensions respectively.

We find that the equations obtained may all be expressed in the form

$$
s u^{\prime \prime}+a u^{\prime}+\bar{b} u+\bar{c} u^{2}=0
$$

where the constant a takes the positive values $\frac{1}{2}, 1, \frac{3}{2}$ and 2 .

In a subsequent paper (Billigheimer 1967-8) the behaviour of solutions of equations of type (1.2) as $s \rightarrow \infty$ with the parameter $a>\frac{1}{2}$ are discussed in greater detail. The behaviour of the solutions as $s \rightarrow 0$ will be examined in detail in a sequel.

1.1. Invariance properties. The equation

$$
\square^{2} u=b u+c u^{2}
$$

is invariant under three main classes of transformations: Displacements, rotations and proper Lorentz transformations. Solutions of (1.11) satisfying an ordinary differential equation may then have the independent variable transformed according to these transformations and will still satisfy the same ordinary differential equation.

In the succeeding work we may take the sign of the constants $b, c$ in equation (1.11) to be positive without loss of generality. For the equation (1.11) is invariant urider the simultaneous transformations

$$
\grave{b}=-\bar{b}, \quad u=\bar{u}+\bar{b} / c ; \quad c=-\bar{c}, \quad u=-\bar{u} .
$$

Similarly in the case of equation (1.2) we need only consider the case $s>0$, as the equation is invariant under the simultaneous tra sformations

$$
s=-\bar{s}, \quad u=-\bar{u}-\bar{b} / \bar{c} .
$$

Throughout this paper we distinguish between the parameter $c$ in equations (1.1), (1.11) and the constant velocity of light $c$ in the expression for $\square^{2}$ in (1.2).

1.2. One-dimensional case. We consider first the one-dimensional case of equation (1.11)

$$
u_{x x}-u_{t \imath} / c^{2}=b u+c u^{2} .
$$

We try to find functions $g(x, t)$ such that a solution $u$ may be a function of the single variable $g$ only. Assume $g$ is such a function and $u=u(g)$. Then Eq. (1.21) becomes

$$
u^{\prime \prime}\left(g_{x}^{2}-\frac{1}{c^{2}} g_{t}^{2}\right)+u^{\prime}\left(g_{x x}-\frac{1}{c^{2}} g_{t u}\right)=b u+c u^{2}
$$

where the primes indicate differentiation with respect to $g$.

We require that

$$
g_{x}^{2}-\frac{1}{c^{2}} g_{t}^{2}=h(g)
$$

and

$$
g_{x x}-\frac{1}{c^{2}} g_{t t}=k(g)
$$

where $h, k$ are functions of $g$. 
Differentiating (1.23) with respect to $x$ and $t$ and substituting in (1.24) we obtain the relation

$$
\frac{g_{x t}}{g_{x} g_{t}}=\frac{h^{\prime}(g)-k(g)}{h(g)}=H(g), \text { say. }
$$

Writing this as

$$
\frac{\partial}{\partial x}\left(\log g_{\imath}\right)=\frac{\partial}{\partial x} \int^{0} H\left(g_{1}\right) d g_{1}
$$

we obtain by integration

$$
g_{t}=\exp \left[p(t)+\int^{0} H\left(g_{1}\right) d g_{1}\right]
$$

where $p(t)$ is an arbitrary function of $t$.

Again writing this as

$$
\frac{\partial}{\partial t} \int^{0}\left\{\exp \left[-\int^{o \cdot} H\left(g_{1}\right) d g_{1}\right]\right\} d g_{2}=\frac{\partial}{\partial t} \int^{t} \exp \left[p\left(t_{1}\right)\right] d t
$$

we integrate to obtain

$$
\int^{0}\left\{\exp \left[-\int^{o s} H\left(g_{1}\right) d g_{1}\right]\right\} d g_{2}=\int^{t} \exp \left[p\left(t_{1}\right)\right] d t_{1}+q(x)
$$

where $q(x)$ is an arbitrary function of $x$.

Solving this relationship for $g$, we obtain

$$
g=F[P(t)+Q(x)]
$$

where $F$ is some function

$$
P(t)=\int^{t} \exp \left[p\left(t_{1}\right)\right] d t_{1}, \quad Q(x)=q(x) .
$$

We may now obviously choose as the fundamental variable simply

$$
g=P(t)+Q(x) \text {. }
$$

We note that this makes $g_{x t}=0$ and hence using (1.25)

$$
k(g)=h^{\prime}(g) \text {. }
$$

To obtain the form of $P(t), Q(x)$ we substitute from (1.26) in (1.23) obtaining

$$
\left(\frac{d Q}{d x}\right)^{2}-\frac{1}{c^{2}}\left(\frac{d P}{d t}\right)^{2}=h[P(t)+Q(x)]=h(g) .
$$

Differentiating with respect to $t$, we obtain

$$
\begin{gathered}
-\frac{1}{c^{2}} \cdot 2 \frac{d P}{d t} \cdot \frac{d^{2} P}{d t^{2}}=\frac{d h}{d g} \cdot \frac{d P}{d t}, \\
\frac{d P}{d t}=0 \quad \text { or } \quad-\frac{2}{c^{2}} \frac{d^{2} P}{d t^{2}}=\frac{d h}{d g} .
\end{gathered}
$$

Similarly differentiating with respect to $x$ we obtain

$$
\frac{d Q}{d x}=0 \text { or } 2 \frac{d^{2} Q}{d x^{2}}=\frac{d h}{d g} .
$$


Ignoring for the moment the space- or time-independent cases we deduce that

$$
\frac{1}{c^{2}} \frac{d^{2} P}{d t^{2}}=-\frac{d^{2} Q}{d x^{2}}=\text { constant }=2 \alpha \text { say }
$$

Hence

$$
P=\alpha c^{2} t^{2}+\beta_{1} t+\gamma_{1}, \quad Q=-\alpha x^{2}+\beta_{2} x+\gamma_{2}
$$

where the $\beta_{i}, \gamma_{i}$ are constants and we obtain

$$
g=\alpha\left(c^{2} t^{2}-x^{2}\right)+\beta_{1} t+\beta_{2} x+\gamma
$$

where $\gamma=\gamma_{1}+\gamma_{2}$.

Substituting into (1.22) we obtain the equation for $u(g)$ accordingly

$$
u^{\prime \prime}(-4 \alpha g+\delta)+u^{\prime}(-4 \alpha)=b u+c u^{2}
$$

where $\delta=\beta_{2}^{2}-\beta_{1}^{2} / c^{2}+4 \alpha \gamma$.

Thus if Eq. (1.21) has a solution $u$ which is a function of the single variable $g(x, t)$ then $g$ must be of the form given by (1.28). This condition, which as can be scen includes the time-independent and space-independent cases, is the condition sought for the partial differential equation (1.21) to reduce to an ordinary differential equation.

1.3. Equation types. The equations (1.29) may be divided into two types. Type 1: Reducible type. If we take $\alpha=0$ in (1.28), (1.29) we obtain the equation

$$
\delta u^{\prime \prime}=b u+c u^{2}
$$

where

$$
g=\beta_{1} t+\beta_{2} x+\gamma, \quad \delta=\beta_{2}^{2}-\beta_{1}^{2} / c^{2} .
$$

This equation is free of explicit dependence on $g$ ind may be reduced to first order and solved explicitly in terms of elliptic functions.

Taking $\beta_{1}=c, \beta_{2}=0$ we obtain the space-independent case

$$
u^{\prime \prime}=-b u-c u^{2}
$$

where $u=u(g)=u(c t+\gamma)$ and similarly taking $\beta_{1}=0, \beta_{2}=1$ we obtain the onedimensional time-independent case

$$
u^{\prime \prime}=b u+c u^{2}
$$

where $u=u(g)=u(x+\gamma)$.

Using the Lorentz-invariance properties of Eq. (1.11) we now obtain the planewave and three-dimensional cases which satisfy the same equation. Thus we have the equation

$$
u^{\prime \prime}=-b u-c u^{2}
$$

where

$$
u=u(g)=u(c t+\gamma)
$$

or

$$
u=u(g)=u\left[c \beta\left(t-v x / c^{2}\right)+\gamma\right]
$$

or

$$
u=u(g)=u\left[c \beta\left(t-(v(l x+m y+n z)) / c^{2}\right)+\gamma\right]
$$


where

$$
\beta=\left(1-v^{2} / c^{2}\right)^{-1 / 2}, \quad l^{2}+m^{2}+n^{2}=1
$$

and the equation

$$
u^{\prime \prime}=b u+c u^{2}
$$

where

$$
u=u(g)=u(x+\gamma)
$$

or

$$
u=u(g)=u[\beta(x-v t)+\gamma],
$$

or

$$
u=u(g)=u[l x+m y+n z+\gamma]
$$

or

$$
u=u(g)=u[\beta(l x+m y+n z-v t)+\gamma] .
$$

By the substitution $s= \pm g^{2}$ respectively the Eqs. (1.31), (1.32) are transformed into the equation

$$
4 s u^{\prime \prime}+2 u^{\prime}+b u+c u^{2}=0
$$

for $s \gtrless 0$ respectively.

In this form their relation to equations of Type 2 which we shall now consider becomes evident.

Type 2: Irreducible type. If we take $\alpha \neq 0$ in (1.28), (1.29) we may put

$$
s=g-\delta / 4 \alpha=\alpha\left[c^{2}(t+A)^{2}-(x+B)^{2}\right]
$$

where

$$
A=\beta_{1} / 2 \alpha c^{2}, \quad B=-\beta_{2} / 2 \alpha .
$$

Choosing $\alpha=1$ and taking the constants $A=B=0$ we then obtain Eq. (1.29) in the form

$$
4 s u^{\prime \prime}+4 u^{\prime}+b u+c u^{2}=0
$$

where $s=c^{2} t^{2}-x^{2}$.

1.4. Circularly and spherically symmetric cases. We derive in this section equations of Type 2 for the three-dimensional spherically symmetric case.

The corresponding equations in the two-dimensional circularly symmetric case are obtained in a precisely parallel way.

We take the three-dimensional spherically symmetric form of Fq. (1.11)

$$
u_{r r}+\frac{2}{r} u_{r}-\frac{1}{c^{2}} u_{t t}=b u+c u^{2}
$$

where $r^{2}=\left(x-x_{0}\right)^{2}+\left(y-y_{0}\right)^{2}+\left(z-z_{11}\right)^{2}$.

Proceeding similarly to the preceding work, we require a solution $u(g)$ where $g=g(r, t)$. This function $g$ must satisfy simultaneously the equations

$$
g_{r}^{2}-\frac{1}{c^{2}} g_{t}^{2}=h(g)
$$


and

$$
g_{r r}+\frac{2}{r} g_{r}-\frac{1}{c^{2}} g_{t t}=k(g)
$$

where $h(g), k(g)$ are functions of $g$.

Differentiating (1.42) with respect to $r$ and $t$ and substituting in the second equation, we obtain

$$
h^{\prime}-h \cdot \frac{g_{r t}}{g_{r} g_{t}}+\frac{2}{r} g_{r}=k .
$$

We shall confine ourselves to cases where $g_{r t}=0$, obtaining

$$
g=P(t)+Q(r) \text {. }
$$

Then we have from (1.44)

$$
\begin{gathered}
\frac{2}{r} g_{r}=k(g)-h^{\prime}(g)=K(g), \\
\frac{d g}{K(g)}=\frac{r}{2} d r .
\end{gathered}
$$

Integrating we obtain

$$
\int \frac{d g}{K(g)}=r^{2}+P(t)
$$

which we may solve to obtain

$$
g=g\left[r^{2}+P(t)\right]
$$

We may take simply

$$
g=r^{2}+P() .
$$

To obtain the form of $P(t)$ we substitute from (1.45) in (1.42) obtaining

$$
4 r^{2}-\frac{1}{c^{2}} \frac{d P^{2}}{d t}=h\left[r^{2}+P(t)\right]=h(g) .
$$

Differentiating with respect to $t$, we obtain

$$
-\frac{1}{c^{2}} \cdot 2 \frac{d P}{d t} \frac{d P}{d t^{2}}=\frac{d h}{d g} \cdot \frac{d P}{d t} .
$$

Hence either

$$
\text { (a) } \quad-\frac{2}{c^{2}} \frac{d^{2} P}{d t^{2}}=\frac{d h}{d g}
$$

or

$$
\text { (b) } \quad \frac{d P}{d t}=0
$$

Differentiating similarly with respect to $r$, we obtain

Hence

$$
8 r=\frac{d h}{d g} \cdot 2 r,
$$

$$
\frac{d h}{d g}=4 \text {. }
$$


We consider firstly case (a) and we have

$$
-\frac{2}{c^{2}} \cdot \frac{d^{2} P}{d t^{2}}=\frac{d h}{d g}=4
$$

Hence $P=-c^{2} t^{2}+\beta t+\gamma$.

Hence $g=r^{2}-c^{2} t^{2}+\beta t+\gamma$.

Then we obtain from (1.42), (1.43) the equation for $u$

$$
u^{\prime \prime}(4 g+\delta)+u^{\prime} \cdot 8-b u-c u^{2}=0 .
$$

where $\delta=-4 \gamma-\beta^{2} / c^{2}$.

Putting $s=-(g+\delta / 4)=-r^{2}+c^{2}\left(t-\beta / 2 c^{2}\right)^{2}$ and taking the constant $\beta=0$, we obtain the equation in the form

$$
4 s u^{\prime \prime}+8 u^{\prime}+b u+c u^{2}=0
$$

where $s=c^{2} t^{2}-r^{2}=c^{2} t^{2}-x^{2}-y^{2}-z^{2}$ say.

In the two-dimensional case, we obtain analogously

$$
4 s u^{\prime \prime}+6 u^{\prime}+b u+c u^{2}=0
$$

where $s=c^{2} t^{2}-r^{2}=c^{2} t^{2}-x^{2}-y^{2}$ say.

Considering now case (b) above we have

Hence

$$
d P / d t=0
$$

$$
P=\gamma \text {. }
$$

Hence $g=r^{2}+\gamma$.

Then we obtain the equation

$$
u^{\prime \prime}(4 g-4 \gamma)+u^{\prime} \cdot 6-b u-c u^{2}=0 .
$$

Putting $s=-g+\gamma=-r^{2}$ we obtain the equation in the form

$$
4 s u^{\prime \prime}+6 u^{\prime}+b u+c u^{2}=0
$$

where $s=-r^{2}=-x^{2}-y^{2}-z^{2}$.

We note that this equation is of the same form (1.47) as in the case where

$$
s=c^{2} t^{2}-x^{2}-y^{2} \text {. }
$$

In two dimensions we similarly obtain the equation

$$
4 s u^{\prime \prime}+4 u^{\prime}+b u+c u^{2}=0
$$

where $s=-r^{2}=-x^{2}-y^{2}$.

This equation is of the same form (1.34) as in the case discussed in Sec. 1.3, where

$$
s=c^{2} t^{2}-x^{2}
$$

We note that we may state the reducible first cases of Sec. 1.3 in an analogous way. For, as we have seen, Eqs. (1.31), (1.32) of Sec. 1.3 may be written in the form

$$
4 s u^{\prime \prime}+2 u^{\prime}+b u+c u^{2}=0
$$

where $s=c^{2} t^{2}$ and $s=-x^{2}$.

We consider in the following sections solutions of the two main types of equation obtained, the cases $a=\frac{1}{2}$ and $a>\frac{1}{2}$ of an equation of the form 


$$
s u^{\prime \prime}+a u^{\prime}+\bar{b} u+\bar{c} u^{2}=0
$$

where we write $\bar{b}=\frac{1}{4} b, \bar{c}=\frac{1}{4} c$.

2. Solutions of the equation $s u^{\prime \prime}+\frac{1}{2} u^{\prime}+\bar{b} u+\bar{c} u^{2}=0$. We consider in $t b_{3}$ section the equation

$$
s u^{\prime \prime}+\frac{1}{2} u^{\prime}+\bar{b} u+\bar{c} u^{2}=0 .
$$

We need only discuss the behaviour of the solution of the equation for positive values of $s$ as (2.1) is invariant under the simultaneous transformations $s=-\bar{s}$, $u=-\bar{u}-\bar{b} / \bar{c}$.

Setting $t=s^{1 / 2}$ in (2.1) we obtain the equation

$$
\ddot{u}+b u+c u^{2}=0 \quad(\cdot=d / d t)
$$

where $b=4 \bar{b}, c=4 \bar{c}$.

In this form we see that the equation, which is free of explicit dependence on the independent variable $t$, may be directly integrated a first time. The solutions may be readily discussed at this stage, or alternatively we can find an explicit expression for the solution in terms of Jacobian elliptic functions.

One of the constants of integration is a "homology constant" $h$, since for any solution $y=f(t)$, we have a corresponding solution $y=f(t+h)$.

Kurdgelaidze (1954) in the third section of his paper has obtained similar plane wave solutions for the equation

$$
\square^{2} u=b u+c u^{3}
$$

in which the nonlinearity is cubic instead of quadratic as in our case.

2.1. Solutions of the Equation $\ddot{u}+b u+c u^{2}=0$. We consider solutions of

$$
\ddot{u}+b u+c u^{2}=0
$$

determined by initial conditions $u=u_{0}, \dot{u}=0$ at $t=0$ and also solutions unbounded at the origin.

We find that for $-b / c<u_{0}<b / 2 c$ we obtain oscillatory solutions about $u=0$, while for $u_{0}<-b / c$ and $u_{0}>b / 2 c$ we have solutions becoming negatively unbounded for finite $t$.

There is a transition case $u_{0}=b / 2 c$ in which the solution tends asymptotically to the limit $-b / c$, as well as the trivial solutions $u \equiv 0, u \equiv-b / c$.

Integrating Eq. (2.11) we obtain

$$
\frac{1}{2} \dot{u}^{2}=\frac{1}{2} b\left(u_{0}^{2}-u^{2}\right)+\frac{1}{3} c\left(u_{0}^{3}-u^{3}\right) .
$$

We can exhibit the solution behaviour of (2.11) by means of the graph in the "phaseplane" $u-\dot{u}$ (Fig. 1). The arrows indicate the sense of description of the curves as $t$ increases.

Integrating (2.12) we obtain

$$
\begin{aligned}
t & =\int_{u_{0}}^{u}\left\{\frac{2}{3} c\left(u_{0}-v\right)\left[\left(\frac{1}{2} u_{0}+3 b / 4 c+v\right)^{2}+\frac{3}{4}\left(u_{0}+b / 2 c\right)^{2}-b^{2} / c^{2}\right]\right\}^{-1 / 2} d \\
& =\int_{u_{0}}^{u}\left\{\frac{2}{3} c(\alpha-v)(v-\beta)(v-\gamma)\right\}^{-1 / 2} d v
\end{aligned}
$$

where $\alpha, \beta, \gamma$, are functions of $u_{0}$.

We obtain explicit representations for the solutions in terms of Jaco ian elliptic functions by inverting (2.13). 


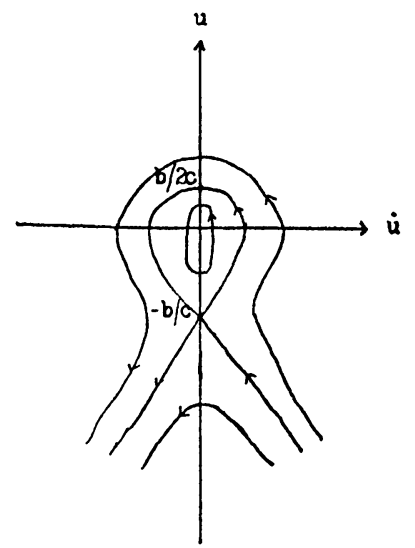

FIG. 1

We thus have apart from the trivial solutions $u \equiv 0, u \equiv-b / c$, the following three main types of solution behaviour.

Type 1: Oscillatory solutions. For $-b / c<u_{0}<b / 2 c$ we have closed curves in the $u-\dot{u}$ plane, corresponding to bounded oscillatory solutions of constant amplitude.

The explicit expression for the solutions for $0<u_{0}<b / 2 c$ is

$$
u=u_{0}-\left(u_{0}-u_{1}\right) \operatorname{sn}\left(\left(\frac{1}{6} c\right)^{1 / 2} \delta t, k\right)
$$

where $\delta^{2}=\alpha-\gamma, k^{2}=(\alpha-\beta)(\alpha-\gamma)$ and $u_{0}=\alpha, u_{1}=\beta$ are the maximum and minimum of the oscillations respectively.

For $-b / c<u_{0}<0$ we have

$$
u=u_{1}-\left(u_{1}-u_{0}\right) c d^{2}\left(\left(\frac{1}{6} c\right)^{1 / 2} \delta t, k\right)
$$

where $\delta^{2}=\beta-\gamma, k^{2}=(\beta-\alpha, \beta-\gamma)$ and $u_{0}=\alpha, u_{1}=\beta$ are the minimum and maximum of the oscillations respectively.

The time of traversal of the closed curves in the $u-\dot{u}$ plane and thus the period of the oscillatory motions is given by $T=2 K(k) /\left(\frac{1}{6} c\right)^{1 / 2} \delta$ (where $K(k)$ is the complete elliptic integral of the first kind), increasing as the amplitude of the motion increases from the linear value $2 \pi(b)^{1 / 2}$ to infinity. This dependence of the period on the amplitude is characteristic of nonlinear oscillations, and is to be contrasted with the independence of period and amplitude in the linear case.

Type 2: Unbounded solutions. For $u_{0}>b / 2 c$ and $u_{0}<-b / c$ the solutions become negatively unbounded for finite $t$.

The explicit expression for the solutions for the cases $u_{0}>b / 2 c$ and $u_{0}<-3 b / 2 c$ may be written as

$$
u=u_{0}+A\left\{\operatorname{cn}\left[\left(\frac{2}{3} c A\right)^{1 / 2} t, k\right]-1\right\} /\left\{c n\left[\left(\frac{2}{3} c A\right)^{1 / 2} t, k\right]+1\right\}
$$

where $A^{2}=3\left(u_{0}^{2}+u_{1}, b / c\right), k^{2}=\frac{1}{2}+3\left(u_{0}+b / 2 c\right) / 4 A$, and for $-3 b / 2 c<u_{0}<-b / c$

$$
u=u_{0}-\left(\gamma-u_{0}\right) s c^{2}\left(\left(\frac{1}{6} c\right) \delta t, k\right)
$$

where $\delta^{2}=\beta-\alpha, k^{2}=(\beta-\gamma)(\beta-\alpha)$. In the limiting case $u_{0}=-3 b_{/}^{\prime} 2 c$ we obtain

$$
u=u_{0} \sec ^{2} \frac{1}{2} b^{1 / 2} t .
$$

By change of origin of $t$ we obtain a corresponding solution negatively unbounded at $t=0$. 
We also have the limiting case of the solution unbounded at the origin which tendis to the value $-b / c$ as $t$ tends to $\infty$.

$$
u=b / 2 c-2 b / 3 c \cdot \operatorname{coth}^{2} \frac{1}{2} b^{1 / 2} t .
$$

Type 3: Transition case. For $u_{0}=b / 2 c$ we have the transition case in which $u$ remains bounded and tends asymptotically to the limiting value $-b / c$ as $t$ tends to $\infty$.

$$
u=b / 2 c-3 b / 2 c \tanh ^{2} \frac{1}{2} b^{1 / 2} t .
$$

3. Solutions of the equation $s u^{\prime \prime}+a u^{\prime}+\bar{b} u+\bar{c} u^{2}=0$. We investigate in this section the solution behaviour of the general equation

$$
s u^{\prime \prime}+a u^{\prime}+\bar{b} u+\bar{c} u^{2}=0
$$

for parameter values $a \geq \frac{1}{2}$ by means of a graphical method [cf. Finkelstein et al (1951) and Mitskevitch (1956)] based upon the consideration in a phase-plane of the energy of a corresponding one-dimensional motion analogous to that considered for the case $a=\frac{1}{2}$ in Sec. 2.2.

Again we need only consider the case $s>0$ as (3.1) is invariant under the simultaneous transformations $s=-\bar{s}, u=-\bar{u}-\bar{b} / \bar{c}$.

For $s>0$ we put $t=s^{1 / 2}$ in the equation (3.1)

$$
s u^{\prime \prime}+a u^{\prime}+\bar{b} u+\bar{c} u^{2}=0
$$

obtaining the equation

$$
\ddot{u}+(2 a-1) \dot{u} / t+b u+c u^{2}=0 .
$$

This may be written in the form

$$
\frac{d}{d u}\left(\frac{1}{2} \dot{u}^{2}+\frac{1}{2} b u^{2}+\frac{1}{3} c u^{3}\right)=-\frac{(2 a-1)}{t} \dot{u}
$$

provided $\dot{u} \neq 0$.

If we regard $u, \dot{u}$ as the position and velocity respectively of a representative particle, then this equation describes a nonconservative one-dimensional motion in which $t$ ("time") occurs explicitly.

The energy for the corresponding conservative motion defined by the Eq. (3.2) with the $1 / t$ term deleted is

$$
\begin{aligned}
E & =\frac{1}{2} \dot{u}^{2}+\frac{1}{2} b u^{2}+\frac{1}{3} c u^{3} \\
& =\frac{1}{2} \dot{u}^{2}+V(u)
\end{aligned}
$$

where $V(u)=\frac{1}{2} b u^{2}+\frac{1}{3} c u^{3}$.

The equilibrium points of the motion defined by $d V / d u=0$ correspond to the special solutions $u=0,-b / c . u=0$ is a stable position of equilibrium, $u=-b / c$ is unstable.

If we consider the $u-\dot{u}$ plane, the point representative of a conservitive motion moves on the curves of constant $E$, as illustrated in Fig. 2 below. The arrowheads indicate the sense of traversal of the curves with increasing $t$ (and $s$ ). These correspond to solutions of Eq. (3.1) with $a=\frac{1}{2}$.

From the equation of motion we derive

$$
\frac{d E}{d u}+\frac{(2 a-1)}{t} \dot{u}=0
$$


or

$$
\frac{d E}{d t}+\frac{(2 a-1)}{t} \dot{u}^{2}=0
$$

Hence for cases $a>\frac{1}{2}, d E / d t$ is always negative for $t>0$, and the representative point of an actual motion always moves inward across lines of constant $E$. Such a trajectory, wherever it starts, must terminate at either $u=0$ or $u=-b / c$ or go off to infinity negatively, depending upon the initial values $u_{0}, \dot{u}_{0}$ and the initial time $t_{0}$ at which these are taken. The slope of the curves in the $u-\dot{u}$ plane is given by

$$
\frac{d u}{d \dot{u}}=\frac{\dot{u}}{\ddot{u}}=\frac{\dot{u} t}{(2 a-1) \dot{u}+t\left(b u+c u^{2}\right)} .
$$

Representative curves that may be expected are indicated dotted in Fig. 2 below.

If we regard the solutions as classified according to the initial value $u_{0}$ at $s=0$, we might expect to be able to summarize the solution behaviour as $s \rightarrow+\infty$ in the following theorem:

TheOREm I. There exists a number $\Lambda \geq b / 2 c$ such that if the solutions of (3.1) with $a \geq \frac{1}{2}$ satisfy initial conditions $u=u_{0}, \dot{u}=0$, at $s=0$, we have for $s>0$ :

(i) The solutions remain bounded and oscillate about the value $u=0$ with diminishing amplitude as $s$ tends to $+\infty$ if $-b / c<u_{0}<\Lambda$, with diminishing amplitude if $a>\frac{1}{2}$.

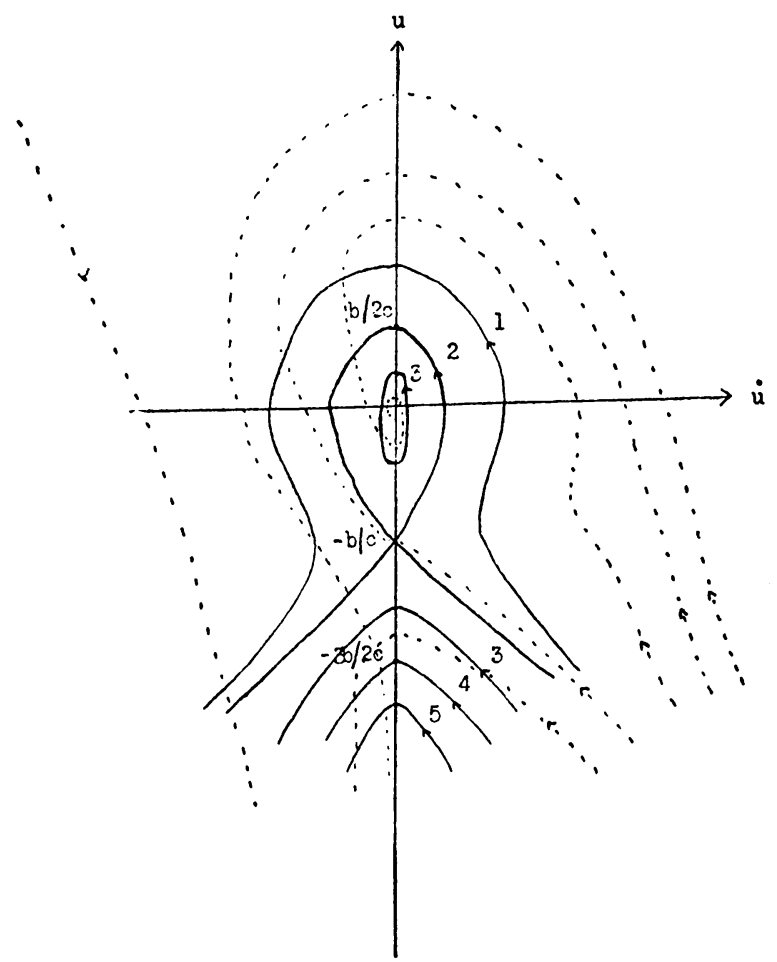

FIG. 2

- Conservative Motion $\left(a=\frac{1}{2}\right)$.

-Actual Motion $\quad\left(a>\frac{1}{2}\right)$

$$
\begin{aligned}
& \text { For curves } 1 \quad: E>E_{0} \\
& 2 \quad: E=E_{0}=b^{3} / 6 c^{2} \\
& 3,4,5: E<E_{0}
\end{aligned}
$$


(ii) For $u_{0}<-b / c$ the solutions tend monotonically to $-\infty$ as s increases.

(iii) For $u_{0}>\Lambda$ the solutions tend monotonically to $-\infty$ as $s$ increases.

(iv) For $u_{0}=\Lambda$ we have the transition case where solutions tend asymptoticaliy to $-b / c$ as $s$ tends to $+\infty$.

We may alternatively classify the solution behaviour by considering varying initial slopes $u_{0}^{\prime}$ at a point $s=s_{0}>0$ with $u=u_{0}, u^{\prime}=u_{0}^{\prime}$.

The behaviour may thus be summarized as follows:

Theorem II. There exists a number $P$ and a number $Q(P<Q)$ such that, if the solutions of $E q$. (3.1) with $a \geq \frac{1}{2}$ satisfy initial conditions $u=u_{0}, u^{\prime}=u_{0}^{\prime}$ at $s=s_{0}>0$, we have for $s>s_{0}$ :

(i) The solutions are bounded and oscillatory about $u=0$, tending to 0 as $s \rightarrow \infty$ for $P<u_{0}^{\prime}<Q$.

(ii) For $u_{0}^{\prime}<P$ the solutions tend ultimately monotonically to $-\infty$ as $s$ increases.

(iii) For $u_{0}^{\prime}>Q$ the solutions tend ultimately monotonically to $-\infty$ as $s$ increases.

(iv) For $u_{0}^{\prime}=P$ and $u_{0}^{\prime}=Q$ we have the transition cases in which $u$ tends ultimately monotonically to $u=-b / c$ as $s \rightarrow \infty$.

The solution behaviour in the case of equations with $a<\frac{1}{2}$ may be similarly discussed. The equation with $a=\frac{1}{2}$ corresponding to the case of conservative motions can be integrated directly in terms of elliptic functions. The oscillatory solutions here have fixed amplitude and period depending on the amplitude. They also provide planewave solutions of the Klein-Gordon equation mentioned earlier:

$$
\square^{2} u=b u+c u^{2} \text {. }
$$

In the case of the nonlinear equation with the parameter $b=0$ asymptotically oscillatory solutions are not found. The solutiori behaviour is thus essentially different from that for the equation with $b \neq 0, c \neq 0 \mathrm{wl}$ ' $\mathrm{ch}$ we have discussed.

Acknowledgments. I am indebted to Professc: C. A. Hurst, University of Adelaide, for suggesting this topic and for his valuable suggestions and criticism.

I also wish to express my thanks to the Directors of General Motors-Holdens Pty. Ltd. and to the Council of the School of General Studies, Australian National University, for the award of a Postgraduate Research Fellowship.

\section{ReFerences}

1. C. E. Billigheimer, Solutions of a non-linear differential equation I, Proc. Camb. Phil. Soc. (1967-8)

2. F. Cap, Consequences of a nonlinear theory of nuclear forces, Nuovo Cimento Suppl, 807-812, (1956)

3. W. Heisenberg, Mesonenerzengung als Stosswell enproblem, Z. Phys. 133, 65-79, (1952)

4. C. A. Hurst, An example of a divergent perturbation expansion in field theory, Proc. Cambridge Philos. Soc. 48, 625-639 (1952)

5. D. F. Kurdgelaidze, Nonlinear scattering in electrodynamics and mesondynamics, Vestn. Moskow Univ. 9, 8, 81-90, (1954)

6. A. Petermann, Renormalization dans les séries divergentes, Helv. Phys. Acta 26, 291-299 (1953)

7. L. I. Schiff, Nonlinear meson theory of nuclear forces, I: Neutral scalar meson with pu.nt-contact repulsion, Pliys. Rev. 84, 1-9 (1951)

8. J. J. Stoker, Nonlinear vibrations, Interscience, New York, 1950

9. W. 'i hirring, Nichtlineare Terme in Meson-Gleichungen, Z. Natur-forsch. 7a, 63-66 (1952)

10. W. Thirring, On the divergence of perturbation theory for quantised fields, Helv. Phys. Acta. 26, 35- 52 (1953)

11. R. Utiyama and T. Imamura, Difficulty of divergence of the perturbation method in quani!" in field theory, Progr. Theor. Phys. 9, 431-454 (1963) 